\title{
A randomized prospective study comparing acute toxicity, compliance and objective response rate between simultaneous integrated boost and sequential intensity-modulated radiotherapy for locally advanced head and neck cancer
}

\author{
Akanksha Grover ${ }^{1}$, Tej Prakash Soni ${ }^{1}$, Nidhi Patni ${ }^{1}$, Dinesh Kumar Singh ${ }^{1}$, Naresh Jakhotia ${ }^{1}$, Anil Kumar Gupta ${ }^{2}$, \\ Lalit Mohan Sharma ${ }^{3}$, Shantanu Sharma ${ }^{4}$, Ravindra Singh Gothwal ${ }^{4}$ \\ ${ }^{1}$ Department of Radiation Oncology, Bhagwan Mahaveer Cancer Hospital and Research Centre, Jaipur, Rajasthan, India \\ ${ }^{2}$ Department of Surgical Oncology, Bhagwan Mahaveer Cancer Hospital and Research Centre, Jaipur, Rajasthan, India \\ ${ }^{3}$ Department of Medical Oncology, Bhagwan Mahaveer Cancer Hospital and Research Centre, Jaipur, Rajasthan, India \\ ${ }^{4}$ Department of Radiotherapy, S.M.S Medical College and Hospital, Jaipur, Rajasthan, India
}

Received: December 6, 2020

Revised: January 15, 2021

Accepted: January 22, 2021

Correspondence:

Tej Prakash Soni

Department of Radiation Oncology,

Bhagwan Mahaveer Cancer Hospital

and Research Centre, Jaipur,

Rajasthan, India

Tel: +91-141-2700107

Fax: +91-141-2702021

E-mail: drtejprakashsoni@yahoo.co.in ORCID:

https://orcid.org/0000-0003-4925-4928
Purpose: Intensity-modulated radiotherapy (IMRT) provides higher dose to target volumes and limits the dose to normal tissues. IMRT may be applied using either simultaneous integrated boost (SIB-IMRT) or sequential boost (SEQ-IMRT) technique. The objectives of this study were to compare acute toxicity and objective response rates between SIB-IMRT and SEQ-IMRT in patients with locally advanced head and neck cancer.

Materials and Methods: Total 110 patients with locally advanced carcinoma of oropharynx, hypopharynx, and larynx were randomized equally into the two arms (SIB-IMRT vs. SEQ-IMRT). Patients in SIB-IMRT arm received dose of 66 Gy in 30 fractions, 5 days a week, over 6 weeks. SEQ-IMRT arm's patients received 70 Gy in 35 fractions over 7 weeks. Weekly concurrent cisplatin chemotherapy was given in both arms. Patients were assessed for acute toxicities during the treatment and for objective response at 3 months after the radiotherapy.

Results: Grade 3 dysphagia was significantly more with SIB-IMRT compared to SEQ-IMRT (72\% vs. $41.2 \% ; p=0.006$ ) but other toxicities including mucositis, dermatitis, xerostomia, weight-loss, incidence of nasogastric tube intubation and hospitalization for supportive management were similar in both the arms. Patients in SIB-IMRT arm showed better treatment-compliance and had significantly less treatment-interruption compared to SEQ-IMRT arm $(p=0.028)$. Objective response rates were similar in both the arms $(\mathrm{p}=0.783)$.

Conclusion: Concurrent chemoradiation with SIB-IMRT for locally advanced head and neck cancer is well-tolerated and results in better treatment-compliance, similar objective response rates, comparable incidence of mucositis and higher incidence of grade 3 dysphagia compared to SEQ-IMRT.

Keywords: Simultaneous integrated boost, Sequential intensity-modulated radiotherapy, Toxicity, Compliance 


\section{Introduction}

Head and neck cancer is a significant global public health problem. Overall, 57.5\% of global head and neck cancers occur in Asia [1]. In India, head and neck cancer accounts for $30 \%$ of all cancer burden and majority of these patients present with locally advanced stages [2]. Tobacco consumption habits such as bidi smoking, tobacco chewing and cigarette smoking are primarily responsible for the high prevalence of these cancers. Treatment of locally advanced head and neck cancer is a clinical challenge and requires aggressive and concerted measures. Radiotherapy with concurrent chemotherapy is the standard curative treatment strategy for the locally advanced head and neck cancer. The ultimate aim of radiation therapy is the maximum local control of the tumor with minimal toxicity. Introduction of three-dimensional conformal radiotherapy (3D-CRT) allowed irradiating the target volumes more precisely with better sparing of surrounding healthy tissues. Advent of intensity-modulated radiotherapy (IMRT) facilitated even more conformity in dose shaping, providing higher dose to target volumes, further limiting the dose to organs at risk thus leading to less toxicity $[3,4]$. IMRT is a modern form of conformal radiotherapy and it is of greater value for target volumes with complex shapes with close proximity to radiosensitive organs at risk (OAR) and normal tissues. IMRT can be applied by using either sequential boost intensity-modulated radiotherapy (SEQ-IMRT) or simultaneous integrated boost intensity-modulated radiotherapy (SIB-IMRT) technique. SEQ-IMRT consists of two phases with shrinking field approach, in first phase large volumes with the gross tumor and the elective nodal region with clinical target volume (CTV) are irradiated, followed by the cone-down plan or sequential boost in second phase to irradiate the gross tumor only. SIB-IMRT has a single treatment plan for the entire course of the treatment with different radiation doses and intensities appropriate for gross tumor volume (GTV) and elective nodal region including CTV. Mohan et al. [5] introduced the term "simultaneous integrated boost" to describe the delivery of different doses per fraction to different target regions including prophylactic lower dose to CTV and curative higher dose to GTV. SEQ-IMRT uses a conventional 1.8-2 Gy per fraction throughout the entire course of radiation treatment with shrinking field approach, while SIB-IMRT delivers 2.2 Gy per fraction to the gross tumor and boost volume and a 1.8 Gy per fraction to the elective volume in the same treatment session. SIB-IMRT and SEQ-IMRT both techniques are being used widely in current practice. However, clinical studies and randomized trials comparing SIB-IMRT and SEQ-IMRT for locally advanced head and neck cancer are scarce and the relative efficacy and toxicities of each technique remain unexplored. In this prospective, randomized study we aimed to compare the acute toxicities and objective response rates between SIB-IMRT and SEQ-IMRT in patients with locally advanced carcinoma of oropharynx, hypopharynx and larynx.

\section{Materials and Methods}

It was a randomized, open label trial between SIB-IMRT and SIQ-IMRT. The protocol and informed consent form were reviewed and approved by the Institutional Review Board of Bhagwan Mahaveer Cancer Hospital and Research Centre, Jaipur (No. BMH/2019/2556). The inclusion criteria were newly diagnosed, histologically proven locally advanced, the American Joint Committee on Cancer (AJCC 8th edition) stage III, IVA carcinoma of oropharynx, hypopharynx and larynx patients with Karnofsky Performance Status (KPS) more than $70 \%$. All the eligible patients fulfilling the inclusion criteria were approached and their clinical history, thorough clinical examination and relevant investigations were recorded. Proper counseling about the concurrent chemoradiotherapy treatment and the trial arms was done to every participant. Informed consent was taken before enrolling them in the study.

As suggested by the results of a retrospective study by Vlacich et al. [6] a sample size of 100 patients was needed to detect $27 \%$ difference in the incidence of grade 3 or worse dysphagia between SIB-IMRT (82\%) and SEQ-IMRT (55\%) assuming an " $\alpha$ " error of 0.05 and a " $\beta$ " error of 0.20 . Assuming that $10 \%$ of patients to be inevaluable (due to lost to follow-up), a total of 110 patients were calculated to be randomized in our study (55 patients in each arm). Linear variables were summarized as mean and standard deviation and were analyzed by using unpaired t-test. Nominal/categorical variables were described as proportions (\%) were analyzed by chisquare test/Fischer exact test. Ordinal variables were presented as median and range were analyzed by using Mann-Whitney test. The p-value less than 0.05 were taken as significant. Total 110 patients with locally advanced carcinoma of oropharynx, hypopharynx and larynx were randomized equally into two arms (SIB-IMRT and SEQ-IMRT) by computer generated random number table method. Target volumes were delineated in accordance with the International Commission on Radiation Units and Measurements (ICRU) Report 62 [7]. GTV were delineated by contouring gross visible or palpable tumor and enlarged or suspicious lymph nodes identified by clinical examination, endoscopy and CT scan or MRI scan imaging. GTV with margin for subclinical microscopic malignant disease was contoured as CTV-high risk (CTV-HR). CTV-HR was delineated as individualized expansions around GTV based on location and specific at-risk sites of subclinical disease, but generally involved a margin of 1.0-1.5 cm around the GTV. For involved nodal levels, the entire level were included in CTV-HR. Low risk areas with po- 
tential subclinical microscopic disease such as ipsilateral and contralateral uninvolved neck nodal groups were contoured and delineated as CTV-low risk (CTV-LR). CTV delineation was done as per the RTOG and TROG consensus guidelines and routinely included bilateral level II-IV lymph nodes [8,9]. If involved at presentation, or clinically indicated (large primary lesion or N2b, N2c or N3 nodal disease), level I and/or level $\mathrm{V}$ lymph nodes were also included in CTV. CTV was edited to exclude natural barriers to disease spread. The planning target volume (PTV) was delineated to provide a margin around the CTV to compensate for the uncertainties of radiotherapy planning, treatment delivery, patients set-up and tissue deformation. An isotropic expansion of $5 \mathrm{~mm}$ was added around the CTV to define each respective PTV-high risk (PTV-HR) and PTVlow risk (PTV-LR). The PTV-HR and PTV-LR were cropped $2 \mathrm{~mm}$ inside the body contour automatically by the treatment planning system. Normal tissue and OAR dose-constraints were prescribed as per the Quantitative Analyses of Normal Tissue Effects in the Clinic (QUANTEC) guidelines [10]. Dose volume objectives (dose constraints) for OAR were prescribed as spinal cord $D_{\max }<44 \mathrm{~Gy}$, brain stem $D_{\max }<54 \mathrm{~Gy}$, mandible $\mathrm{D}_{\max }<70 \mathrm{~Gy}$, and parotid glands $\mathrm{D}_{\text {mean }}<26 \mathrm{~Gy}$, oral cavity $\mathrm{D}_{\text {mean }}<40 \mathrm{~Gy}$. IMRT plan objectives were to achieve no more than $20 \%$ of any PTV volumes could receive $>110 \%$ of its prescribed dose, no more than $1 \%$ of any PTV volume would receive $<93 \%$ of the prescribed dose and no more than $1 \%$ or $1 \mathrm{~mL}$ of the tissue outside the PTV would receive $>110 \%$ of the dose prescribed to the primary target. The most important objective was to keep maximum dose to spinal cord and brain stem below 45 Gy and $54 \mathrm{~Gy}$, respectively. The second priority for OAR was to reduce the mean dose to parotids where possible to less than $26 \mathrm{~Gy}$. Patients in SIB-IMRT arm received dose of 66 Gy to PTV-HR (2.2 Gy per fraction) and 54 Gy to PTV-LR (1.8 Gy per fraction) in 30 fractions, 5 days a week, over 6 weeks. Patients in SEQ-IMRT arm received dose of $50 \mathrm{~Gy}$ in 25 fractions in 5 weeks (2 Gy per fraction) to PTV-HR and PTV-LR in phase I followed by 20 Gy in 10 fractions in 2 weeks (2 Gy per fraction) to PTV-HR in phase II (combined total dose in phase I and II was 70 Gy in 35 fractions in 7 weeks). For both the arms, IMRT was planned on Eclipse version 15 Treatment Planning System (TPS) with RapidArc technique (Varian Medical System, Palo Alto, CA, USA) by using a double arc with 6-MV photon beam. Concurrent weekly cisplatin chemotherapy (total $6 \mathrm{cy}-$ cles) to dose of $40 \mathrm{mg} / \mathrm{m}^{2}$ was given to the patients in both arms during radiotherapy. Standard oral, medical and supportive treatment was provided to the patients of both arms. All participants were assessed weekly during chemoradiotherapy treatment for acute toxicities including oral mucositis, dysphagia, dermatitis, xerostomia, weight-loss, requirement of nasogastric tube intubation for feeding and admission in the hospital for supportive care man- agement. Grading of oral mucositis, dysphagia, dermatitis, and xerostomia was done as per the Common Terminology Criteria for Adverse Events (CTCAE) version 4.03. Weekly complete blood count and renal function test were done during chemoradiotherapy. $\mathrm{Ob}-$ jective response assessment was done after three months of chemoradiotherapy treatment with the Response Evaluation Criteria in Solid Tumor (RECIST 1.1) criteria by clinical examination, endoscopy, and CT scan or MRI.

\section{Results}

From February 2019 to March 2020, total 110 patients were enrolled prospectively and randomized equally into two arms (SIB-IMRT and SEQ-IMRT). Fifty-five patients were enrolled in each arm. The CONSORT diagram showing flowchart of the treatment is presented in the Fig. 1. Five patients in SIB-IMRT arm and four patients in SEQ-IMRT arm defaulted before completion of the treatment, so they were excluded from the final analysis. Total 101 patients were included in the final analysis; 50 patients received SIB-IMRT and 51 patients received SEQ-IMRT. Mean age of patients was 56.1 years (range, 47.4 to 64.8 years). Ninety-five were male $(94.1 \%)$ and six patients (5.9\%) were female. The site of primary tumor was oropharynx, larynx, and hypopharynx in 71 (70.29\%), 16 (15.8\%), and 14 patients (13.86\%), respectively. Baseline patient and tumor characteristics (sex, age, primary tumor site, stage, co-morbidity, and history of tobacco consumption) were similar among both the study groups (Table 1). Seventy-seven patients (76.23\%) re-

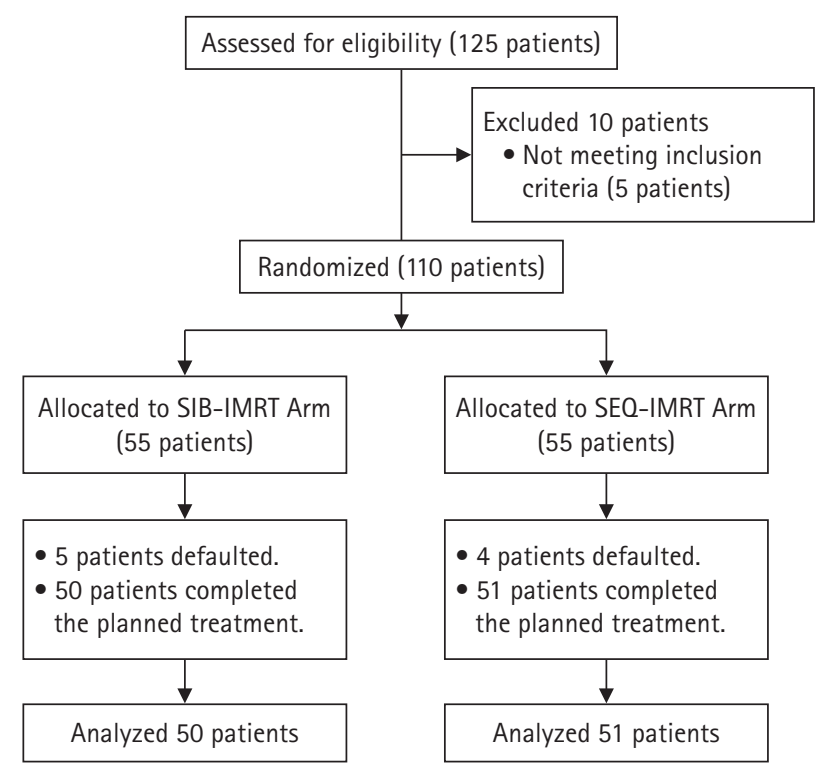

Fig. 1. The CONSORT diagram showing the flow of the patients' selection. SEQ-IMRT, sequential intensity-modulated radiotherapy, SIB-IMRT: simultaneously integrated boost intensity-modulated radiotherapy. 
Table 1. Baseline patient characteristics between SIB-IMRT and SEQ-IMRT arms

\begin{tabular}{|c|c|c|c|}
\hline Characteristic & SIB-IMRT $(n=50)$ & SEQ-IMRT $(n=51)$ & $p$-value \\
\hline Mean age (yr) & 56.3 & 56.04 & 0.002 \\
\hline Gender & & & 0.621 \\
\hline Male & $46(92.0)$ & $49(96.1)$ & \\
\hline Female & $4(8.0)$ & $2(3.9)$ & \\
\hline History of tobacco consumption & 45 (90.0) & $46(90.1)$ & 0.143 \\
\hline Co-morbidity & & & 0.967 \\
\hline Diabetes & $2(4.0)$ & $3(5.9)$ & \\
\hline Ischemic heart disease & $3(6.0)$ & $3(5.9)$ & \\
\hline Hypertension & $6(12.0)$ & $7(13.7)$ & \\
\hline T stage & & & 0.302 \\
\hline $\mathrm{T} 1$ & $2(4.0)$ & $3(5.9)$ & \\
\hline T2 & $20(40.0)$ & $18(35.3)$ & \\
\hline $\mathrm{T} 3$ & $15(30.0)$ & 19 (37.2) & \\
\hline $\mathrm{T} 4$ & $13(26.0)$ & $11(21.6)$ & \\
\hline $\mathrm{N}$ stage & & & 0.406 \\
\hline No & $8(16.0)$ & $10(19.6)$ & \\
\hline $\mathrm{N} 1$ & $14(28.0)$ & $12(23.5)$ & \\
\hline N2 & $28(56.0)$ & $29(56.9)$ & \\
\hline Primary site & & & 0.121 \\
\hline Oropharynx & $36(72.0)$ & 35 (68.6) & \\
\hline Larynx & $7(14.0)$ & $9(17.6)$ & \\
\hline Hypopharynx & $7(14.0)$ & $7(13.7)$ & \\
\hline Stage grouping & & & 0.133 \\
\hline Stage III & $25(50.0)$ & $29(56.86)$ & \\
\hline Stage IVA & $25(50.0)$ & $22(43.13)$ & \\
\hline 6 cycles weekly chemotherapy received & $38(76.0)$ & $39(76.5)$ & 0.956 \\
\hline
\end{tabular}

Values are presented as number of patients (\%).

SIB-IMRT, simultaneous integrated boost intensity-modulated radiotherapy; SEQ-IMRT, sequential boost intensity-modulated radiotherapy.

ceived full course of planned 6 cycles of weekly concurrent cisplatin chemotherapy. Thirty-eight patients (76\%) in SIB-IMRT arm versus 39 patients (76.47\%) in SEQ-IMRT arm received 6 cycles of concurrent chemotherapy $(p=0.956)$ (Table 1$)$. All patients received a median 6 cycles of concurrent chemotherapy (mean, 5.6 cycle; range, 3 to 6 cycle). There was no significant difference in terms of number of chemotherapy cycles received between both the arms (Table 1). Radiation dose and volume characteristics for the oral cavity and esophagus are reported in Table 2. Oral cavity and esophagus OAR's mean volume, mean dose, maximum dose $\left(D_{\text {max }}\right)$ were not statistically different between the two arms (Table 2).

\section{Acute toxicity assessment}

The 5.9\%, 37.7\%, and 56.4\% of total patients developed grade 1, grade 2, and grade 3 dysphagia, respectively. Patients in SEQ-IMRT arm had significantly lower incidence of grade 3 dysphagia (41.2\% vs. $72 \%$ ) compared to SIB-IMRT ( $p=0.006$ ) (Table 3). There were no significant differences in incidence of mucositis, dermatitis, xe-
Table 2. Dosimetric comparison between SIB-IMRT and SEO-IMRT arm

\begin{tabular}{llll}
\hline OAR & SIB-IMRT & SEQ-IMRT & p-value \\
\hline Oral cavity & & & \\
$\quad$ Mean volume $(\mathrm{mL})$ & 61.02 & 65.25 & 0.404 \\
Mean dose $(\mathrm{Gy})$ & 38.5 & 37.9 & 0.312 \\
$\mathrm{D}_{\max }(\mathrm{Gy})$ & 68.8 & 70.2 & 0.085 \\
V40 $(\mathrm{mL})$ & 22.44 & 26.76 & 0.653 \\
Esophagus & & & \\
Volume $(\mathrm{mL})$ & 9.2 & 7.3 & 0.087 \\
Mean dose (Gy) & 23.4 & 26.1 & 0.202 \\
$\mathrm{D}_{\max }($ Gy) & 53 & 51 & 0.097 \\
\hline
\end{tabular}

SIB-IMRT, simultaneous integrated boost intensity-modulated radiotherapy; SEQ-IMRT, sequential boost intensity-modulated radiotherapy; $O A R$, organs at risk.

rostomia, weight-loss, incidence of hospitalization for supportive management and requirement of nasogastric tube intubation for feeding (Table 3).

The $8.9 \%, 48.5 \%$, and $42.6 \%$ patients developed grade 1, grade 
Table 3. Acute toxicity profile comparison between SIB-IMRT and SEQ-IMRT arm

\begin{tabular}{|c|c|c|c|}
\hline Toxicity & SIB-IMRT $(n=50)$ & SEQ-IMRT $(n=51)$ & $p$-value \\
\hline Mucositis & & & 0.425 \\
\hline Grade 2 & $27(54.0)$ & $22(43.1)$ & \\
\hline Grade 3 & $20(40.0)$ & $23(45.1)$ & \\
\hline Dysphagia & & & 0.006 \\
\hline Grade 1 & $1(2.0)$ & $5(9.8)$ & \\
\hline Grade 2 & $13(26.0)$ & $25(49)$ & \\
\hline Grade 3 & $36(72.0)$ & $21(41.2)$ & \\
\hline Xerostomia & & & 0.566 \\
\hline Grade 1 & $36(72.0)$ & $33(64.7)$ & \\
\hline Grade 2 & $14(28.0)$ & $18(35.3)$ & \\
\hline Dermatitis & & & 0.429 \\
\hline Grade 1 & $16(32.0)$ & $21(41.2)$ & \\
\hline Grade 2 & $29(58.0)$ & $23(45.1)$ & \\
\hline Grade 3 & $5(10.0)$ & $7(13.7)$ & \\
\hline Hospitalization for supportive treatment & $20(40.0)$ & $16(31.3)$ & 0.072 \\
\hline Mean weight-loss $(\mathrm{kg})$ & 5.04 & 5.6 & 0.439 \\
\hline Requirement of nasogastric tube intubation & $20(40.0)$ & $15(29.4)$ & 0.130 \\
\hline Mean treatment interruption (gap in radiotherapy) (day) & 2.80 & 4.78 & 0.028 \\
\hline
\end{tabular}

Values are presented as number of patients (\%).

SIB-IMRT, simultaneous integrated boost intensity-modulated radiotherapy; SEQ-IMRT, sequential boost intensity-modulated radiotherapy.

2 , and grade 3 oral mucositis, respectively. Twenty patients (40\%) versus 23 patients (45.1\%) developed grade 3 mucositis in SIB-IMRT and SEQ-IMRT arms, respectively ( $p=0.425$ ).

There was no significant difference in incidence of grade 3 oral mucositis between both the arms (Table 3). In SIB-IMRT arm, 16 (32\%), 29 (58\%), and 5 patients (10\%) developed grade 1, grade 2, and grade 3 dermatitis, respectively. In SEQ-IMRT arm, 21 (41.2\%), $23(45.1 \%)$, and 7 patients (13.7\%) developed grade 1, grade 2, and grade 3 dermatitis, respectively $(p=0.429)$. There was no significant difference in incidence of dermatitis between both the arms (Table 3).

The patients in SIB-IMRT arm had better treatment-compliance compared to SEQ-IMRT arm. In SIB-IMRT arm, mean treatment-interruption (gap in radiotherapy) was 2.8 days (range, 1 to 10 days) whereas in SEQ-IMRT arm it was 4.78 days (range, 1 to 8 days) (p $=0.028$ ) (Table 3).

In SIB-IMRT arm, 36 (72\%) and 14 patients (28\%) developed grade 1 and grade 2 xerostomia, respectively. In SEQ-IMRT arm, 33 $(64.7 \%)$ and 18 patients (35.3\%) developed grade 1 and grade 2 xerostomia, respectively $(p=0.566)$. There was no significant difference in incidence of xerostomia between both the arms (Table 3).

In SIB-IMRT arm, mean weight-loss of patients was $5.04 \mathrm{~kg}$ (range, 1 to $11 \mathrm{~kg}$ ) while in SEQ-IMRT it was $5.6 \mathrm{~kg}$ (range, 1 to $12.5 \mathrm{~kg})(p=0.439)$. There was no significant difference in incidence of weight-loss between both the arms (Table 3).

Total 35 patients $(34.6 \%)$ required nasogastric tube intubation for feeding. Twenty patients (40\%) in SIB-IMRT arm and 15 patients (29.4\%) in SEQ-IMRT arm required nasogastric intubation ( $p$ $=0.130$ ) (Table 3). Although more patients in SIB-IMRT arm required nasogastric tube intubation compared to SEQ-IMRT but it was not statistically significant.

Total 36 patients (35.64\%) required indoor admission in the hospital for supportive treatment and management of dysphagia. Twenty patients (40\%) in SIB-IMRT arm versus 16 patients (31.37\%) in SEQ-IMRT arm required admission in the hospital ( $p=$ 0.072) (Table 3). Although more patients in SIB-IMRT arm required hospitalization for supportive care compared to SEQ-IMRT but it was not statistically significant.

\section{Objective response assessment}

Objective response to the treatment evaluation was done after 3 months of completion of the treatment. Seventy-three patients (72.27\%) had complete response, 27 patients (26.73\%) had partial response, and one patient had progressive disease. Objective response rate were comparable in both the treatment arms ( $p=$ 0.783) (Table 4).

\section{Discussion and Conclusion}

In this study we evaluated and compared the outcomes and acute toxicities between SIB-IMRT and SEQ-IMRT for locally advanced head and neck cancer patients. In our knowledge, probably this is 
Table 4. Objective tumor response rate comparison between SIB-IMRT and SEQ-IMRT arm

\begin{tabular}{lccc}
\hline Objective response & SIB-IMRT & SEQ-IMRT & p-value \\
\hline Complete response & $36(72)$ & $37(72.54)$ & 0.783 \\
Partial response & $13(26)$ & $14(28)$ & \\
Progressive disease & $1(2)$ & $0(0)$ & \\
\hline
\end{tabular}

Values are presented as number of patients (\%).

SIB-IMRT, simultaneous integrated boost intensity-modulated radiotherapy; SEQ-IMRT, sequential boost intensity-modulated radiotherapy.

the first randomized trial comparing SIB-IMRT and SEQ-IMRT in non-nasopharyngeal, locally advanced head and neck cancer patients. Overall results of our study suggest that SIB-IMRT and SEQ-IMRT treatment in locally advanced head and neck cancer along with concurrent chemotherapy are equivalent with respect to treatment outcomes. Although higher incidence of grade 3 dysphagia was observed with the SIB-IMRT compared to SEQ-IMRT however, it did not translate into statistically significant increased weight-loss or nasogastric intubation. Other acute toxicities, including mucositis, dermatitis and xerostomia, were comparable in both the study arms.

The observed lower incidence of dysphagia in SEQ-IMRT arm in our study might be attributed to the reduced radiation exposure of the lower neck and pharyngeal constrictors from 6 weeks in SIB-IMRT to 5 weeks in SEQ-IMRT. Two Gy per fraction for 5 weeks to lower neck and pharyngeal constrictors in SEQ-IMRT might have produced lesser severe dysphagia compared to $1.8 \mathrm{~Gy}$ per fraction for 6 weeks in SIB-IMRT. More grade 3 mucositis (45\% vs. 40\%; p $=0.425$ ) and less nasogastric tube intubation (29.4\% vs. $40 \% ; p$ $=0.439$ ) probably resulted in increased weight-loss (mean $5.6 \mathrm{~kg}$ vs. $5.04 \mathrm{~kg} ; \mathrm{p}=0.439$ ) in SEQ-IMRT arm compared to SIB-IMRT arm, although these differences between the both arms were statistically not significant.

Diligent treatment-compliance is crucial and important management strategy. Unplanned treatment interruptions and prolonged radiation treatment time are associated with poor treatment outcomes. Langendijk et al. [11] in a study of oral cancer patients concluded that overall treatment time is the most important prognostic factor. The authors observed the locoregional control worsened approximately $9 \%$ with each additional week in prolongation of overall treatment [11]. In our study, patients in SIB-IMRT arm showed better treatment-compliance and had significantly less interruption in radiation treatment compared to SEQ-IMRT arm. In SIB-IMRT arm, mean treatment-interruption (gap in radiotherapy) was 2.8 days whereas in SEQ-IMRT arm it was 4.78 days ( $p=$ 0.028). Less number of radiation fractions (30 fractions compared to 35 fractions) and 1 week shorter treatment schedule ( 6 weeks vs. 7 weeks) in SIB-IMRT might be provided better logistic support to patients compared to SEQ-IMRT and it could be the reason for better compliance and less treatment interruptions. As majority of patients have severe dysphagia and treatment interruption in last 2 weeks of the radiotherapy, 1 week shorter overall treatment time in SIB-IMRT arm might be increased the compliance.

In a comparative, retrospective study of 209 patients of locally advanced head and neck cancer, between SIB-IMRT versus SEO-IM$R T$, there were no significant differences in local, regional, or distant recurrence-free survival [6]. In this study, grade 3 or 4 dysphagia ( $81 \%$ vs. $55 \%)$ and dermatitis (78\% vs. $58 \%$ ) were significantly higher in the SIB-IMRT compared to SEQ-IMRT ( $p<0.001$ and $p<$ 0.012) [6]. There were no significant differences in the rate of gastrostomy tube placement and relative weight-loss between SIB-IMRT and SEQ-IMRT. These results are comparable to ours study with comparable objective response rate between both the treatment technique and higher incidence of grade 3 dysphagia in SIB-IMRT arm.

In a population-based propensity score based analysis, Kuo et al. [12] compared SIB-IMRT and SEQ-IMRT in 200 patients of carcinoma oropharynx and hypopharynx patients. The hazard ratio (HR) of death between SIB-IMRT and SEQ-IMRT was 1.23 ( $p=0.29$ ). The authors concluded that the survival outcome is comparable between both the techniques.

In a retrospective comparative study between SIB-IMRT versus SEO-IMRT in 398 locally advanced head and neck cancer patients, the authors [13] found similar rates of 2-year locoregional control and overall survival. Patients in SIB-IMRT had lower rates of acute grade 3 mucositis (22.4\% vs. $36.7 \%$; $p=0.0001)$, dermatitis $(7.5 \%$ vs. $20 \% ; p=0.0001$ ), and feeding tube placement (44\% vs. $51 \%$; $p=0.0001)$ compared to SEQ-IMRT arm [13]. In our study also there was no difference in objective response rate between both the arms with comparable incidence of mucositis, dermatitis and nasogastric tube placement.

Songthong et al. [14], in a prospective randomized study of 112 patients of carcinoma nasopharynx, compared SIB-IMRT and SEQ-IMRT and found no significant difference in acute toxicities or short-term treatment outcomes. The incidence of grade 3 acute toxicities; mucositis (15.4\% vs. $13.6 \%$; $p=0.788)$, dysphagia (9.6\% vs. $9.1 \% ; p=1.000)$, xerostomia ( $9.6 \%$ vs. $7.6 \% ; p=0.748$ ) and one year overall survival (95.8\% vs. $95.5 \% ; p=0.472)$ or progression free survival ( $98 \%$ vs. $90.2 \% ; p=0.069$ ) were not statistically different between SEQ-IMRT versus SIB-IMRT [14].

In a randomized study of 209 patients of carcinoma nasopharynx, the authors [15] compared SIB-IMRT with SEQ-IMRT. There were no statistically significant differences in the incidence of grade 3-4 acute toxicities between the two techniques (59.8\% in SEQ-IMRT vs. $58.9 \%$ in SIB-IMRT; $p=0.892$ ). The progression-free 
survival (72.7\% vs. $73.4 \% ; p=0.488)$ and overall survival $(86.3 \%$ vs. $83.6 \% ; p=0.938$ ) were also similar in both the techniques [15]. Results of our study are comparable to this study with no difference in treatment outcomes and mucositis between both the arms.

In a retrospective comparative study between SIB-IMRT versus SEO-IMRT in 107 patients of carcinoma nasopharynx, the authors [16] found no significant difference in 5-year overall survival between both the techniques ( $80.9 \%$ vs. $80.5 \%$; $p=0.568)$ and 5 -year progression-free survival (73.3\% vs. $74.4 \% ; p=0.773)$. In a meta-analysis of total 1,049 patients from seven studies to compare the treatment outcomes and acute toxicity of the SIB-IMRT versus SEQ-IMRT in head and neck cancer patients, the authors [17] found no significant difference in the overall survival $(H R=0.94$; $p=0.71)$, progression free survival $(H R=1.03 ; p=0.79)$, locoregional recurrence-free survival $(H R=0.98 ; p=0.91)$, and distance metastasis-free survival $(H R=0.87 ; p=0.63)$. There was no significant difference in acute toxicities such as dermatitis, mucositis, dysphagia, xerostomia between the SIB-IMRT and SEQ-IMRT groups. The authors concluded that SIB-IMRT and SEQ-IMRT groups had similar treatment outcomes and acute toxicities.

In general, SIB-IMRT and SEQ-IMRT both have their own specific advantages and disadvantages concerning to radiobiology and radiation treatment planning. SIB-IMRT may increase the biologically equivalent dose (BED) delivery to the tumor with higher dose per fraction (>2 Gy per fraction) and shorter overall treatment time. Increasing BED, higher dose per fraction and shorter overall radiation treatment time may result in improved tumor control in locally advanced head and neck cancer patients.

Dosimetric comparison studies have also reported better target coverage, more conformal dose distribution and sparing of OAR with SIB-IMRT compared to SEQ-IMRT in head and neck cancer patients $[18,19]$. SIB-IMRT technique provides more practical and efficient way of radiation treatment planning with less uncertainties to the treatment planning, dose delivery, plan implementation and patient set-up on the couch as it uses a single plan for the entire course of radiotherapy [5]. Disadvantage of SIB-IMRT includes that acceleration of radiotherapy by SIB-IMRT may increase the incidence of severe acute toxicity such as dysphagia, dermatitis and mucositis and worsen the treatment-compliance [20]. SEQ-IMRT is more time consuming as it involves the summation of two or more phased treatment plans, which can produce more uncertainty in dose distribution and delivery. It is of more concern and challenging for the centers with higher number of patients for the treatment per day, by the limited staff and resources as compared to single plan with SIB-IMRT. SIB-IMRT uses a single radiotherapy plan throughout the entire course of the treatment with fewer ra- diation fractions, which reduces overall treatment time and overall expenses incurred by the patients on the treatment. It results in reduction of workload for radiation workers and shorter waiting list of patients for the treatment in busy radiotherapy centers. Unavailability and inaccessibility of modern radiotherapy linear accelerator machines as well as long waiting list of patients for teletherapy is a crucial problem for low-middle income countries like India. The International Atomic Energy Agency has included India along with the poorest sub-Saharan African countries with less than one teletherapy machine per million people $[21,22]$. The World Health Organization recommends at least one teletherapy unit per million populations $[21,22]$. With this recommendation, the minimum required number of teletherapy units is approximately 1,250 as against the presently available 550 units in India, making a huge shortfall of nearly 700 units $[21,22]$. One week shorter treatment schedules in SIB-IMRT compared to SEQ-IMRT can reduce the treatment-cost and the long waiting list of patients for radiation therapy in low resource counties like India.

In summary, our study shows that concurrent chemoradiation with SIB-IMRT for locally advanced head and neck cancer is well-tolerated and results in better treatment-compliance, comparable objective response rate, comparable mucositis, and higher but acceptable incidence of dysphagia compared to SEQ-IMRT. To our knowledge, our study is the first randomized trial to date exploring the acute toxicity profile and objective response rate between SIB-IMRT and SEQ-IMRT as two distinct treatment approaches in non-nasopharyngeal, locally advanced head and neck cancer patients. The strengths of our study includes similar chemotherapy regimens, similar dose-volume constraints and prospective grading of toxicity by a single, experienced radiation oncologist for both the study arms to minimizes the potential for intra- and inter-observer variability in these more subjective values. There are several limitations including it was a single institution based non-blind randomized study with short-term follow-up of patients. A large scale, multicentric, blinded randomized trial with long-term follow-up of patients is recommended to validate the results.

In conclusion, this study shows that concurrent chemoradiation with SIB-IMRT for locally advanced head and neck cancer is well-tolerated and results in better treatment-compliance, similar objective response rate, comparable mucositis and higher but acceptable incidence of dysphagia compared to SEQ-IMRT. Although higher rate of grade 3 radiation induced dysphagia was observed with the SIB-IMRT compared to SEQ-IMRT however, it did not translate into increased weight-loss or more nasogastric tube intubation. SIB-IMRT is a feasible and time sparing treatment technique with encouraging locoregional results and controlled side effects for locally advanced head and neck cancer patients. 


\section{Conflict of Interest}

No potential conflict of interest relevant to this article was reported.

\section{Acknowledgements}

We are thankful to our medical physicist team including Mr. T. Senthil Kumar, Mr. T. Natrajan, Mr. Rajkamal, Ms. Ashna Jenny for their excellent performance and main role in radiotherapy treatment planning, radiation treatment implementation and quality-assurance process.

\section{References}

1. Gupta B, Johnson NW, Kumar N. Global epidemiology of head and neck cancers: a continuing challenge. Oncology 2016;91:1323.

2. Kulkarni MR. Head and neck cancer burden in India. Int J Head Neck Surg 2013;4:29-35.

3. van der Veen J, Nuyts S. Can intensity-modulated-radiotherapy reduce toxicity in head and neck squamous cell carcinoma? Cancers (Basel) 2017;9:135.

4. Tubiana M, Eschwege F. Conformal radiotherapy and intensity-modulated radiotherapy: clinical data. Acta Oncol 2000; 39:555-67.

5. Mohan R, Wu Q, Manning M, Schmidt-Ullrich R. Radiobiological considerations in the design of fractionation strategies for intensity-modulated radiation therapy of head and neck cancers. Int J Radiat Oncol Biol Phys 2000;46:619-30.

6. Vlacich G, Stavas MJ, Pendyala P, Chen SC, Shyr Y, Cmelak AJ. A comparative analysis between sequential boost and integrated boost intensity-modulated radiation therapy with concurrent chemotherapy for locally-advanced head and neck cancer. Radiat Oncol 2017;12:13.

7. Landberg T, Chavaudra J, Dobbs J, Gerard JP, Hanks G, Horiot JC, et al. ICRU Report 62: prescribing, recording and reporting photon beam therapy (supplement to ICRU Report 50). J ICRU [Internet]. 1999 [cited 2021 Feb 11];os32:np. Available from: https:// doi.org/10.1093/jicru/os32.1.Report62.

8. Gregoire V, Evans M, Le QT, et al. Delineation of the primary tumour clinical target volumes (CTV-P) in laryngeal, hypopharyngeal, oropharyngeal and oral cavity squamous cell carcinoma: AIRO, CACA, DAHANCA, EORTC, GEORCC, GORTEC, HKNPCSG, HNCIG, IAG-KHT, LPRHHT, NCIC CTG, NCRI, NRG Oncology, PHNS, SBRT, SOMERA, SRO, SSHNO, TROG consensus guidelines. Radiother Oncol 2018;126:3-24.
9. Gregoire V, Levendag P, Ang KK, et al. CT-based delineation of lymph node levels and related CTVs in the node-negative neck: DAHANCA, EORTC, GORTEC, NCIC, RTOG consensus guidelines. Radiother Oncol 2003;69:227-36.

10. Marks LB, Yorke ED, Jackson A, et al. Use of normal tissue complication probability models in the clinic. Int J Radiat Oncol Biol Phys 2010;76(3 Suppl):S10-9.

11. Langendijk JA, de Jong $M A$, Leemans $C R$, et al. Postoperative radiotherapy in squamous cell carcinoma of the oral cavity: the importance of the overall treatment time. Int J Radiat Oncol Biol Phys 2003:57:693-700.

12. Kuo YH, Liang JA, Wang TC, Juan CJ, Li CC, Chien CR. Comparative effectiveness of simultaneous integrated boost vs sequential intensity-modulated radiotherapy for oropharyngeal or hypopharyngeal cancer patients: a population-based propensity scorematched analysis. Medicine (Baltimore) 2019;98:e18474.

13. Spiotto MT, Weichselbaum RR. Comparison of 3D confromal radiotherapy and intensity modulated radiotherapy with or without simultaneous integrated boost during concurrent chemoradiation for locally advanced head and neck cancers. PLoS One 2014;9:e94456.

14. Songthong AP, Kannarunimit D, Chakkabat $C$, Lertbutsayanukul $C$. A randomized phase II/III study of adverse events between sequential (SEQ) versus simultaneous integrated boost (SIB) intensity modulated radiation therapy (IMRT) in nasopharyngeal carcinoma; preliminary result on acute adverse events. Radiat Oncol 2015;10:166.

15. Lertbutsayanukul C, Prayongrat A, Kannarunimit D, Chakkabat C, Netsawang B, Kitpanit S. A randomized phase III study between sequential versus simultaneous integrated boost intensity-modulated radiation therapy in nasopharyngeal carcinoma. Strahlenther Onkol 2018;194:375-85.

16. Tao H, Wei Y, Huang W, Gai X, Li B. Comparison of long-term survival and toxicity of simultaneous integrated boost vs conventional fractionation with intensity-modulated radiotherapy for the treatment of nasopharyngeal carcinoma. Onco Targets Ther 2016;9:1865-73.

17. Jiang L, Zhang $Y$, Yang Z, Liang F, Wu J, Wang R. A comparison of clinical outcomes between simultaneous integrated boost (SIB) versus sequential boost (SEQ) intensity modulated radiation therapy (IMRT) for head and neck cancer: a meta-analysis. Medicine (Baltimore) 2019;98:e16942.

18. Dogan N, King S, Emami B, Mohideen N, Mirkovic N, Leybovich $L B$, et al. Assessment of different IMRT boost delivery methods on target coverage and normal-tissue sparing. Int J Radiat Oncol Biol Phys 2003;57:1480-91.

19. Fogliata A, Bolsi A, Cozzi L, Bernier J. Comparative dosimetric 
evaluation of the simultaneous integrated boost with photon intensity modulation in head and neck cancer patients. Radiother Oncol 2003;69:267-75.

20. Bourhis J, Sire C, Lapeyre M, et al. Accelerated radiotherapy and/ or concomitant chemotherapy in locally advanced head and neck SCC: results of a GORTEC randomized trial. Radiother Oncol 2008;88(Suppl 2):S121.

21. International Atomic Energy Agency. Setting up a radiotherapy programme: clinical, medical physics, radiation protection and safety aspects. Vienna, Austria: International Atomic Energy Agency; 2008.

22. International Atomic Energy Agency. Availability of radiotherapy: number of radiotherapy machine per million persons [Internet]. Vienna, Austria: International Atomic Energy Agency; 2010 [cited 2021 Feb 11]. Available from: https://www.iaea.org/sites/default/ files/publications/magazines/bulletin/bull52-1/52105810809_0. pdf. 